\title{
Dual Connectedness of Edge-Bicolored Graphs and Beyond
}

\author{
Leizhen $\mathrm{Cai}^{\star}$ and Junjie Ye \\ Department of Computer Science and Engineering, \\ The Chinese University of Hong Kong, Shatin, Hong Kong SAR, China \\ \{lcai, jjye\}@cse.cuhk.edu.hk
}

\begin{abstract}
Let $G$ be an edge-bicolored graph where each edge is colored either red or blue. We study problems of obtaining an induced subgraph $H$ from $G$ that simultaneously satisfies given properties for $H$ 's red graph and blue graph. In particular, we consider DuAlLy CONNECTED INDUCED SUBGRAPH problem - find from $G$ a $k$-vertex induced subgraph whose red and blue graphs are both connected, and DuAL SEPARATOR problem - delete at most $k$ vertices to simultaneously disconnect red and blue graphs of $G$.

We will discuss various algorithmic and complexity issues for DUally Connected Induced Subgraph and Dual Separator problems: NP-completeness, polynomial-time algorithms, W[1]-hardness, and FPT algorithms. As by-products, we deduce that it is NP-complete and $\mathrm{W}$ [1]-hard to find $k$-vertex (resp., $(n-k)$-vertex) strongly connected induced subgraphs from $n$-vertex digraphs. We will also give a complete characterization of the complexity of the problem of obtaining a $k$-vertex induced subgraph $H$ from $G$ that simultaneously satisfies given hereditary properties for $H$ 's red and blue graphs.
\end{abstract}

Keywords: Edge-bicolored graph, dually connected, dual separator.

\section{Introduction}

Edge-colored graphs are fundamental in graph theory and have been extensively studied in the literature, especially for alternating cycles, monochromatic subgraphs, heterchromatic subgraphs and partitions [112]. In this paper, we focus on edge-bicolored graphs — simple undirected graphs $G$ where each edge is uniquely colored by either blue or red, and we use $G_{b}$ and $G_{r}$ to denote the red and blue graphs of $G$ respectively. We are interested in finding an induced subgraph from $G$ that simultaneously satisfies specified properties for its red and blue graphs. In particular, we study the following three closely related problems concerning the fundamental property of being connected for edge-bicolored graphs $G$.

\footnotetext{
* Partially supported by GRF grant CUHK410212 of the Research Grants Council of Hong Kong.
}

E. Csuhaj-Varjú et al. (Eds.): MFCS 2014, Part II, LNCS 8635, pp. 141-152, 2014.

(C) Springer-Verlag Berlin Heidelberg 2014 
- Dually Connected Induced Subgraph: Does $G$ contain exactly $k$ vertices $V^{\prime}$ such that both $G_{b}\left[V^{\prime}\right]$ and $G_{r}\left[V^{\prime}\right]$ are connected?

- Dually Connected Deletion: Does $G$ contain exactly $k$ vertices $V^{\prime}$ such that both $G_{b}-V^{\prime}$ and $G_{r}-V^{\prime}$ are connected?

- Dual Separator: Does $G$ contain at most $k$ vertices $V^{\prime}$ such that both $G_{b}-V^{\prime}$ and $G_{r}-V^{\prime}$ are disconnected?

Related Work: In connection with our dually connected subgraph problems, Gai et al. [7] defined a common connected component of two graphs $G_{1}$ and $G_{2}$ on the same vertex set $V$ as a maximal subset $V^{\prime} \in V$ such that induced subgraphs $G_{1}\left[V^{\prime}\right]$ and $G_{2}\left[V^{\prime}\right]$ are both connected, and they also mentioned three typical applications in computational biology. Using partition refinement to maintain connectivity dynamically, they obtained an algorithm for finding all common connected components in $O\left(n \log n+m \log ^{2} n\right)$ time. For the same problem, Bin-Xuan et al. 2] used their technique of competitive graph search to produce an algorithm with running time $O\left(n+m \log ^{2} n\right)$. We also note that when both $G_{1}$ and $G_{2}$ are paths, the problem of finding all common connected subgraphs coincides with the well studied problem of finding all common intervals of two permutations [18, a problem with many applications.

On the other hand, despite an enormous amount of work on induced subgraph and vertex deletion problems on uncolored graphs, we are unaware of any systematic investigation of the type of problems we study in this paper.

Our Contributions: We study both traditional and parameterized complexities of the above three problems, which has further inspired general induced subgraph problems on edge-bicolored graphs. The following list summarizes our results.

1. Dually Connected Induced Subgraph is NP-complete and W[1]-hard even when both $G_{b}$ and $G_{r}$ are trees, but is solvable in $O\left(n^{2} \alpha\left(n^{2}, n\right)\right)$ time when $G$ is a complete graph, where $\alpha\left(n^{2}, n\right)$ is inverse of Ackermann's function.

2. Dually Connected Deletion is NP-complete and W[1]-hard but admits an FPT algorithm when both $G_{b}$ and $G_{r}$ are trees.

3. Dual Separator is NP-complete.

4. It is NP-complete and W[1]-hard to obtain $k$-vertex (resp. $(n-k)$-vertex) strongly connected induced subgraphs from $n$-vertex digraphs.

5. We give a complete characterization of both classical and parameterized complexities of the INDUCED $\left(\Pi_{b}, \Pi_{r}\right)$-SUBGRAPH problem for hereditary properties $\Pi_{b}$ and $\Pi_{r}$ : Does an edge-bicolored graph $G$ contain a $k$-vertex induced subgraph whose blue and red graphs simultaneously satisfy properties $\Pi_{b}$ and $\Pi_{r}$ respectively?

6. We give FPT algorithms for parametric dual problems of INDUCED $\left(\Pi_{b}, \Pi_{r}\right)$ SuBGRAPH when properties $\Pi_{b}$ and $\Pi_{r}$ admit finite forbidden induced subgraph characterizations.

Notation and Definitions: For a graph $G, V(G)$ and $E(G)$ denote its vertex set and edge set respectively, and $n$ and $m$, respectively, are numbers of vertices 
and edges of $G$. For a subset $V^{\prime} \subseteq V(G), N_{G}\left(V^{\prime}\right)$ denotes the neighbors of $V^{\prime}$ in $V(G)-V^{\prime}$ and $G\left[V^{\prime}\right]$ the subgraph of $G$ induced by $V^{\prime}$. A graph property $\Pi$ is a collection of graphs, and it is hereditary if every induced subgraph of a graph in $\Pi$ also belongs to $\Pi$. It is well-known that $\Pi$ is hereditary iff it has a forbidden induced subgraph characterization.

For an edge-bicolored graph $G=\left(V, E_{b} \cup E_{r}\right), G_{b}=\left(V, E_{b}\right)$ and $G_{r}=\left(V, E_{r}\right)$, respectively, denote the blue graph and red graph of $G$. We say that $G$ is dually connected if both $G_{b}$ and $G_{r}$ are connected, and a dual tree if both $G_{b}$ and $G_{r}$ are trees. A dually connected component of $G$ is a maximal dually connected induced subgraph of $G$. A subset $V^{\prime} \subseteq V(G)$ is a dual separator of $G$ if both $G_{b}-V^{\prime}$ and $G_{r}-V^{\prime}$ are disconnected. We use $\alpha\left(n^{2}, n\right)$ for inverse of Ackermann's function.

Remark: In this paper we require monochromatic subgraphs to be spanning subgraphs of $G$, but for some applications we may disregard isolated vertices in monochromatic subgraphs. For example, we can define $G_{b}=G\left[E_{b}\right]$ or $G_{b}=$ $\left(V_{b}, E_{b}\right)$ with $V_{b} \subseteq V$. Our results in the paper are also valid for these two alternative definitions of monochromatic subgraphs, except our FPT algorithm for Dually Connected Deletion on dual trees (see Problem 11in Section 5).

\section{Dually Connected Induced Subgraphs}

Although all dually connected components in an edge-bicolored graph can be found in $O\left(n+m \log ^{2} n\right)$ time [2, it is surprisingly difficult to determine whether an edge-bicolored graph contains a dually connected induced subgraph on exactly $k$ vertices. We will show that DuAlly Connected Induced SubGraph is solvable in $O\left(n^{2} \alpha\left(n^{2}, n\right)\right)$ time when $G$ is a complete graph, but NP-complete and W[1]-hard when $G$ is a dual tree, i.e., both blue and red graphs of $G$ are trees, which rules out efficient ways to list all common connected subgraphs of two trees. We begin with a lemma for edge-bicolored complete graphs.

Lemma 1. A dually connected edge-bicolored complete graph $G$ contains, for every $4 \leq k \leq n$, a $k$-vertex dually connected induced subgraph.

Proof. For a vertex $v$, if $G-v$ remains dually connected, we can delete $v$ from $G$ and regard the smaller graph as $G$. Therefore, we need only consider the case that $G$ contains a vertex $v$ such that $G-v$ is not dually connected. W.l.o.g., we may assume that $v$ is a cut vertex of $G_{b}$. Since $G_{r}$ is connected, $v$ is not an isolated vertex of $G_{r}$ and hence not adjacent to all vertices of $G_{b}$. Therefore $G_{b}$ has a vertex $x$ such that $d_{G_{b}}(v, x)=2$. Let $y$ be a vertex of $G_{b}-v$ not in the component containing $x$. Then we have $d_{G_{b}}(x, y) \geq 3$.

We now use a breadth-first search from $v$ to obtain $k \geq 4$ vertices $S$, including $\{v, x, y\}$, such that $G_{b}[S]$ is connected. Since $d_{G_{b}[S]}(x, y) \geq d_{G_{b}}(x, y) \geq 3$, we see that in $G_{b}[S]$, no vertex is adjacent to both $x$ and $y$, and hence every vertex is adjacent to at least one of $x$ and $y$ in the complement of $G_{b}[S]$, i.e., graph $G_{r}[S]$. Since $\{x, y\}$ is an edge in $G_{r}[S]$, any pair of vertices in $G_{r}[S]$ has distance at most 3 and hence $G_{r}[S]$ is connected, implying that $G[S]$ is dually connected. 
Theorem 1. Dually Connected Induced Subgraph can be solved in $O\left(n^{2} \alpha\left(n^{2}, n\right)\right)$ time for edge-bicolored complete graphs $G$.

Proof. First we find a largest dully connected component $H$ in $G$. If $G$ itself is dully connected, then set $H$ to $G$. Otherwise, one of $G_{b}$ or $G_{r}$ is disconnected, and $G$ 's dually connected components are equivalent to its maximal strong modules [11, which can be found in linear time by modular decomposition [11. If $k \leq 3$ or $|V(H)|<k$, then the answer is "No"; otherwise the answer is "Yes" by Lemma 1.

Now we discuss how to find a $k$-vertex dully connected subgraph inside $H$. Order vertices of $H$ as $v_{1}, v_{2}, \ldots, v_{h}$ with $h=|V(H)|>k$, and let $V_{i}=\left\{v_{1}, \ldots, v_{i}\right\}$. By Lemma1, we only need to find the smallest index $i>k$ such that $H\left[V_{i}\right]$ is dually connected but $H\left[V_{i-1}\right]$ is not, and then find our required $k$-vertex subgraph inside $H\left[V_{i}\right]$.

For this purpose, we construct $H$ by adding $v_{1}, v_{2}, \ldots, v_{h}$ one by one in this order and, in the process, we use disjoint sets to dynamically maintain components of $H_{b}\left[V_{i}\right]$ and $H_{r}\left[V_{i}\right]$. For the blue graph $H_{b}$ (similar for the red graph $H_{r}$ ), blue sets are components of $H_{b}\left[V_{k}\right]$ initially. In adding vertex $v_{i}$ to $H(i>k)$, we create a blue singleton set $\left\{v_{i}^{b}\right\}$ for vertex $v_{i}$, and for each blue edge $v_{i} v_{j}$ with $j<i$, we merge $\left\{v_{i}^{b}\right\}$ with the blue set containing $v_{j}^{b}$. The procedure stops once there is only one blue set and one red set, i.e., $H\left[V_{i}\right]$ is dually connected but $H\left[V_{i-1}\right]$ is not. Now we can use the proof of Lemma 1 to find a $k$-vertex dually connected subgraph in $O\left(n^{2}\right)$ time. Using standard Union-Find data structure, we can find the required $H\left[V_{i}\right]$ in $O\left(n^{2} \alpha\left(n^{2}, n\right)\right)$ time, which is also an upper bound of our algorithm.

We now introduce a structure called dual 2t-path that will be useful in proving the intractability of Dually Connected Induced Subgraph and also Dually Connected Deletion in the next section. For any $t \geq 3$, a dual $2 t$-path $P^{*}$ is the edge-bicolored graph formed by taking the union of a blue path $P_{b}=v_{1} v_{2} \ldots v_{2 t}$ and red path $P_{r}=v_{2 t} v_{2 t-2} \ldots v_{4} v_{2} v_{2 t-1} v_{2 t-3} \ldots v_{3} v_{1}$ (see Figure 1 for an example). We denote the two ends $v_{1}$ and $v_{2 t}$ of $P^{*}$ by $v^{b}$ and $v^{r}$ respectively.
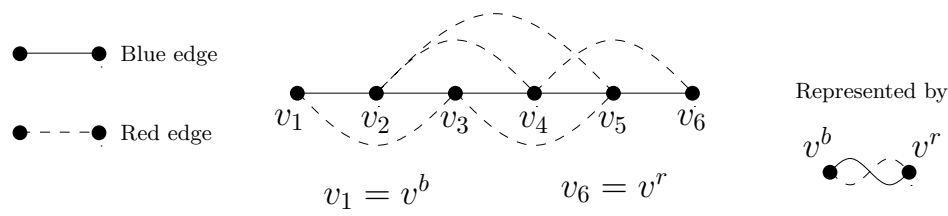

Fig. 1. Dual 2t-path for $t=3$

Lemma 2. In an edge-bicolored graph $G=\left(V, E_{b} \cup E_{r}\right)$, if $V^{*} \subseteq V$ induces a dual 2t-path $P^{*}$ with ends $v^{b}$ and $v^{r}$ such that the only edges between $V^{*}$ and $V-V^{*}$ are blue edges (resp., red edges) between $v^{b}$ (resp., $v^{r}$ ) and $V-V^{*}$, then for any dually connected induced subgraph $G^{\prime}$ of $G$ that contains a vertex in $V^{*}$ and a vertex in $V-V^{*}, G^{\prime}$ must contain all vertices of $V^{*}$. 
Proof. Deleting some but not all vertices in $V^{*}$ of $P^{*}$ will disconnect the blue or red graph of $G^{\prime}$.

Theorem 2. Dually Connected Induced Subgraph is NP-complete and W[1]-hard for dual trees.

Proof. The problem is clearly in NP, and we give a polynomial and FPT reduction from the classical NP- and W[1]-complete CLIQUE problem [8] to prove the theorem. For an instance $(G, k)$ of CLIQUE, we construct an edge-bicolored graph $G^{\prime}$ such that both $G_{b}^{\prime}$ and $G_{r}^{\prime}$ are trees (see Figure 2 for an example):

1. Set $p=k(k-1)$ and create a new vertex $v^{*}$.

2. Replace every vertex $v$ of $G$ by a dual $p$-path $P_{v}^{*}$ with end vertices $v^{b}$ and $v^{r}$, and refer to vertices in $P_{v}^{*}$ as path-vertices. Add blue edge $v^{b} v^{*}$ and red edge $v^{r} v^{*}$.

3. For each edge $e=u v$ of $G$, create edge-vertex $\widetilde{e}$, and replace $e$ by blue edge $u^{b} \widetilde{e}$ and red edge $v^{r} \widetilde{e}$.
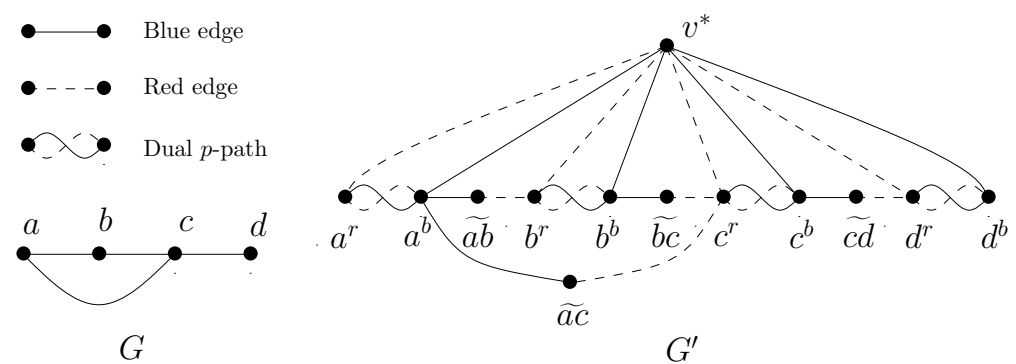

Fig. 2. Construction of $G^{\prime}$ from $G$

It is easy to see that the construction of $G^{\prime}$ takes polynomial time, and that $G_{b}^{\prime}$ and $G_{r}^{\prime}$ are both trees. We claim that $G$ has a $k$-clique iff $G^{\prime}$ has $k^{\prime}=1+k p+p / 2$ vertices $S$ such that $G^{\prime}[S]$ is dually connected.

Assume that $G$ has a $k$-clique $\left\{v_{1}, v_{2}, \ldots, v_{k}\right\}$. Let $S$ be the union of $\left\{v^{*}\right\}$, path-vertices of all $v_{i}$ and edge-vertices of all $v_{i} v_{j}$. The size of $|S|$ is $1+k p+$ $k(k-1) / 2=k^{\prime}$. Since edge-vertex of each $v_{i} v_{j}$ is dually connected to $v^{*}$ through $v_{i}^{b}$ and $v_{j}^{r}, G^{\prime}[S]$ is dually connected.

Conversely, suppose that $G^{\prime}$ contains $k^{\prime}$ vertices $S$ such that $G^{\prime}[S]$ is dually connected. Since all dual $p$-paths are dually connected through $v^{*}, S$ must contain $v^{*}$. Also by Lemma 2, $S$ contains either all or no vertices of any dual $p$-path $P_{v}^{*}$. Therefore $S$ contains path-vertices of at most $k$ dual $p$-paths as $|S|=1+k p+p / 2$. Furthermore, since an edge-vertex $\widetilde{x y}$ is dually connected to $v^{*}$ through both vertices $x^{b}$ and $y^{r}, S$ must contain both $x^{b}$ and $y^{r}$ when $S$ contains an edge-vertex $\widetilde{x y}$. Thus $S$ contains at most $k(k-1) / 2$ edge-vertices. It follows that $S$ contains path-vertices of exactly $k$ dual $p$-paths, and their corresponding vertices in $G$ form a $k$-clique of $G$.

We can regard the complement graph of $G^{\prime}$ in the above proof as a graph with the third color, and obtain the following result to complement Theorem 1 
Corollary 1. Given an edge-tricolored complete graph, it is NP-complete and $W[1]$-hard to find an induced subgraph on exactly $k$ vertices that is connected in every monochromatic graph.

\section{Dual Connectedness by Vertex Deletion}

The intractability of Dually Connected Induced Subgraph calls for an investigation of the parameterized complexity of its dual problem DUALLY CONNECTED Deletion: Can we delete exactly $k$ vertices from an edge-bicolored graph so that the resulting graph is dually connected?

We show that Dually Connected Deletion is also W[1]-hard but becomes FPT for dual trees, which is in contrast to the W[1]-hardness of DUALLY Connected Induced Subgraph on dual trees. Our FPT algorithm uses the following connection with a vertex cover problem that is solvable by the random separation method of Cai, Chan and Chan [4].

Lemma 3. For any dual tree $T$ and $k$ vertices $S$ of $T, T-S$ is a dual tree iff $S$ covers exactly $2 k$ edges.

Proof. Note that both blue and red graphs of $T-S$ are forests, and an $n$ vertex dual tree contains $2(n-1)$ edges. Therefore $T-S$ contains at most $2(n-k-1)=2(n-1)-2 k$ edges, and thus $S$ covers at least $2 k$ edges. This min-max relation implies our lemma.

Theorem 3. Dually Connected Deletion is NP-complete and W[1]-hard, but FPT on dual trees.

Proof. We start with an FPT algorithm for the problem on dual trees $T$. By Lemma 3, it suffices to find $k$ vertices in $T$ that cover exactly $2 k$ edges. We use a modification of the random separation algorithm of Cai, Chan and Chan [4] for finding a subset of vertices to cover exactly $k$ edges.

First, we regard $T$ as an uncolored graph and produce a random black-white coloring for the vertices of $T$. We begin by using black to color all vertices with degree more than $2 k$, and then we randomly and independently color each uncolored vertex by black or white with probability $\frac{1}{2}$. Given a black-white coloring of vertices of $T$, a set $S$ of $k$ vertices is a well-colored solution if

1. $S$ covers exactly $2 k$ edges, and

2. all vertices in $S$ are white and all vertices in $N_{T}(S)$ are black.

Let $V_{w}$ denote the set of white vertices, and refer to connected components of $T\left[V_{w}\right]$ as white components. For a white component $H_{i}$, let $n_{i}$ be the number of vertices in $H_{i}$ and $e_{i}$ the number of edges covered by vertices of $H_{i}$. Then a well-colored solution consists of a collection $\mathcal{H}^{\prime}$ of white components satisfying

$$
\sum_{H_{i} \in \mathcal{H}^{\prime}} n_{i}=k \text { and } \sum_{H_{i} \in \mathcal{H}^{\prime}} e_{i}=2 k .
$$


Therefore we can easily formulate the problem of finding a well-colored solution as a 0-1 knapsack problem, and solve it in $O(k n)$ time using the standard dynamic programming algorithm for the 0-1 knapsack problem. Note that it takes $O(n)$ time to compute all $n_{i}$ and $e_{i}$.

Since a well-colored solution $S$ satisfies $\left|S \cup N_{T}(S)\right| \leq 3 k$, our random blackwhite coloring has probability at least $2^{-3 k}$ to produce a well-colored solution. Therefore when $T$ has a solution, we can find it with probability at least $2^{-3 k}$ in $O(k n)$ time. We can derandomize the algorithm by a family of $(n, 3 k)$-universal sets of size $8^{k} k^{O(\log k)} \log n$ [16], and thus obtain a deterministic FPT algorithm running in time $8^{k} k^{O(\log k)} n \log n$.

For our problem on general edge-bicolored graphs, we give an FPT reduction from the classical W[1]-complete InDEPENDENT SET problem [6 to show W[1]hardness. For an arbitrary instance $(G, k)$ of INDEPENDENT SET, we construct an edge-bicolored graph $G^{\prime}$ from $G$ as follows (see Figure 3 for an example):

1. Replace each edge $u v$ of $G$ by the replacement gadget $H_{u v}$ in Figure 3 .

2. Create a dual $2 k$-path $P^{*}$ with end vertices $v^{b}$ and $v^{r}$, and connect every vertex of $G$ to $v^{b}$ by a blue edge and to $v^{r}$ by a red edge.

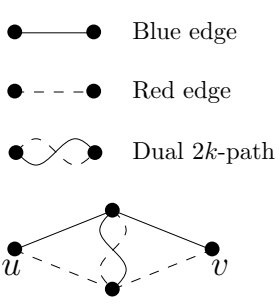

Replacement gadget $H_{u v}$

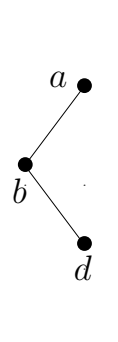

G

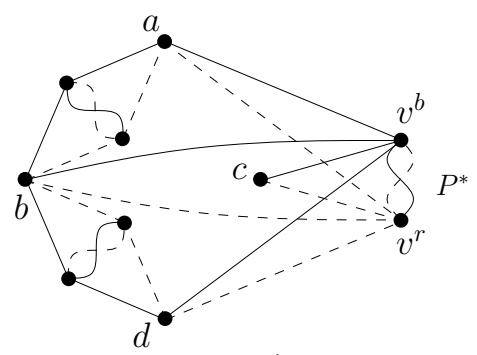

$G^{\prime}$

Fig. 3. Construction of $G^{\prime}$ by using the replacement gadget $H_{u v}$

The construction clearly takes polynomial time, and we show that $G$ has an independent $k$-set iff we can deleting $k$ vertices from $G^{\prime}$ to obtain a dually connected graph.

If $G$ contains an independent set $S$ with $k$ vertices, then for each edge $e$ of $G$, at least one end-vertex, say $v$, of $e$ remains in $G^{\prime}-S$. It is easy to verify that $G^{\prime}-S$ is dually connected as all vertices of $H_{e}$ in $G^{\prime}-S$ are dually connected to $v$, which is dully connected to the dual path $P^{*}$ in Step 2.

Conversely, suppose that $G^{\prime}$ contains $k$ vertices $S$ such that $G^{\prime}-S$ is dually connected. By the property of dual paths (Lemma 22), neither dual $2 k$-path $P^{*}$ nor dual $2 k$-path in any $H_{e}$ contains any vertex from $S$. Therefore all vertices in $S$ are vertices of $G$, and we show that $S$ is an independent set of $G$. For any two vertices $u, v \in S$, if $u v$ is an edge of $G$, then the dual $2 k$-path in $H_{u v}$ is disconnected from $G^{\prime}$ after deleting $S$, contrary to the assumption that $G^{\prime}-S$ is dually connected. Therefore no two vertices in $S$ are adjacent in $G$, and thus $S$ is an independent $k$-set of $G$. 


\section{Dual Separators}

Complimenting dual connectedness, we now consider problems of disconnecting blue and red graphs simultaneously by vertex deletion. In particular, we study Dual Separator and Dual Separator For Two Terminals: Is it possible to disconnect two given vertices $s$ and $t$ in both $G_{b}$ and $G_{r}$ of an edge-bicolored graph $G$ by removing $\leq k$ vertices?

Although minimum separators in uncolored graphs can be found in polynomial time, it is intractable to find minimum-size dual separators in edge-bicolored graphs as we will show that both dual separator problems are NP-complete. However, the parameterized complexity of these two problems remain open.

Theorem 4. Dual Separator For Two Terminals is NP-complete.

Proof. The problem is clearly in NP, and we prove the theorem by a reduction from VERTEX COVER on cubic graphs, whose NP-completeness was established by Garey, Johnson and Stockmeyer [9]. Given a cubic graph $G=(V, E)$, we construct an edge-bicolored graph $G^{\prime}$ as follows (see Figure 4 for an example):

1. Partition edges of $G$ into two bipartite graphs $G_{b}=\left(X_{b}, Y_{b} ; E_{b}\right)$ and $G_{r}=$ $\left(X_{r}, Y_{r} ; E_{r}\right)$, and color all edges of $G_{b}$ blue and all edges of $G_{r}$ red.

2. Introduce two new vertices $s$ and $t$ as terminals.

3. Connect $s$ with every vertex in $X_{b}$ by a blue edge and every vertex in $X_{r}$ by a red edge. Similarly, connect $t$ with every vertex in $Y_{b}$ by a blue edge and every vertex in $Y_{r}$ by a red edge.

4. Turn the above multigraph into a simple graph by subdividing each blue edge incident with $s$ or $t$.

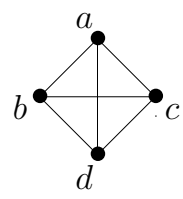

$G$

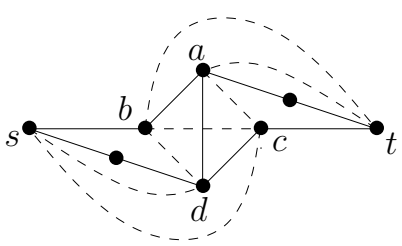

$G^{\prime}$

Fig. 4. In graph $G^{\prime}$, blue edges are solid and red edges are dashed

The above construction takes polynomial time since we can partition edges of any cubic graph $G$ into two bipartite graphs $G_{b}$ and $G_{r}$ in polynomial time by using, for instance, a proper edge 4-coloring of $G$.

In $G^{\prime}$, it is easy to see that every monochromatic $(s, t)$-path goes through some edge of $G$, and every edge of $G$ is contained in some monochromatic $(s, t)$-path of $G^{\prime}$. This clearly implies that for any set $S$ of vertices of $G, S$ is a vertex cover 
of $G$ iff it is a dual $(s, t)$-separator of $G^{\prime}$. Furthermore, we notice that if an $(s, t)$ separator $S^{\prime}$ of $G^{\prime}$ involves some vertices $V^{*}$ used for subdividing blue edges, we can always replace $V^{*}$ by its neighbors in $X_{b} \cup Y_{b}$ to get an $(s, t)$-separator $S$ with $|S| \leq\left|S^{\prime}\right|$. Therefore we can conclude that $G$ admits a vertex cover with $\leq k$ vertices iff $G^{\prime}$ has an $(s, t)$-separator with $\leq k$ vertices, and hence we have a required polynomial reduction.

The above theorem enables us to show the hardness of DUAL SEPARATOR.

\section{Theorem 5. DuAl SEPARATOR is NP-complete.}

Proof. We give a polynomial reduction from Dual Separator For Two TerMINALS by constructing a new graph $G^{\prime \prime}$ from the graph $G^{\prime}$ in the proof of Theorem 4 Let $S_{b}^{\prime}$ (resp., $T_{b}^{\prime}$ ) denote vertices that subdivide blue edges incident with $s$ and $t$ in $G^{\prime}$. To duplicate a vertex $v$, we create a new vertex $v^{\prime}$ and add all blue (resp., red) edges between $v$ and vertices corresponding to $N_{G_{b}^{\prime}}(v)$ (resp., $\left.N_{G_{r}^{\prime}}(v)\right)$. We construct $G^{\prime \prime}$ as follows:

1. Take graph $G^{\prime}$, and duplicate $k$ copies of $s$ and $t$. Denote by $S$ vertex $s$ and its $k$ duplicates, and denote by $T$ vertex $t$ and its $k$ duplicates.

2. Make $k$ duplicates of each vertex in $S_{b}^{\prime}$ (resp., $T_{b}^{\prime}$ ). Denote by $S_{b}^{\prime \prime}$ (resp., $T_{b}^{\prime \prime}$ ) vertices $S_{b}^{\prime}$ (resp., $T_{b}^{\prime}$ ) and all their duplicates.

3. Between $S_{b}^{\prime \prime}$ (resp., $T_{b}^{\prime \prime}$ ) and $T$ (resp., $S$ ), add all possible edges and color them red.

It is easy to see that $G^{\prime}$ has a dual $(s, t)$-separator of size $k$ iff $G^{\prime \prime}$ has a dual $(S, T)$-separator of size $k$. We claim that $G^{\prime \prime}$ has a dual $(S, T)$-separator of size $k$ iff it has a dual separator of size $k$, which will prove the theorem.

A dual $(S, T)$-separator is certainly a dual separator of $G^{\prime \prime}$. Conversely, suppose that $G^{\prime \prime}$ does not have a dual $(S, T)$-separator of size $k$. Then after deleting $k$ vertices $V^{*}$, we will get a graph $G^{*}$ such that $S$ and $T$ are connected in $G_{b}^{*}$ or $G_{r}^{*}$. Note that each vertex in $W=S \cup T \cup S_{b}^{\prime \prime} \cup T_{b}^{\prime \prime}$ has $k+1$ copies, and thus it is useless to delete any vertices in $W$. Hence we can assume that $V^{*} \cap W=\emptyset$. Suppose that $S$ and $T$ are connected in $G_{r}^{*}$. Since $V(G)=X_{r} \cup Y_{r}$, each vertex in $V(G) \cap V\left(G^{*}\right)$ is adjacent to a vertex in $S$ or $T$ with red edges. Furthermore all vertices in $S_{b}^{\prime \prime} \cup T_{b}^{\prime \prime}$ are adjacent to $S$ or $T$ with red edges by Step 3 in constructing $G^{\prime \prime}$, implying that $G_{r}^{*}$ is connected. By a similar argument, we can deduce that $G_{b}^{*}$ is connected when $S$ and $T$ are connected in $G_{b}^{*}$. Thus we have proved this theorem.

\section{Concluding Remarks}

Results on connectedness and separators for edge-bicolored graphs have shown a rich diversity of the complexity of induced subgraph problems on edge-bicolored graphs, which extends an invitation for studying various induced subgraph problems on edge-bicolored graphs. In fact we have obtained a complete characterization of INDUCED $\left(\Pi_{b}, \Pi_{r}\right)$-SUBGRAPH on edge-bicolored graphs for hereditary properties $\Pi_{b}$ and $\Pi_{r}$, and also obtained FPT algorithms for its parametric dual 
problems for properties with finite forbidden induced subgraph characterizations. Furthermore, the work in the paper also enables us to obtain some results for digraphs. Due to space limit, proofs for the following theorems are omitted and will appear in the full paper.

Building on a characterization of Khot and Raman [13 for induced subgraph problems on uncolored graphs and Ramsey's theorem, we completely characterize the complexity of INDUCED $\left(\Pi_{b}, \Pi_{r}\right)$-SUBGRAPH on edge-bicolored graphs for hereditary properties $\Pi_{b}$ and $\Pi_{r}$, which depends on whether $\Pi_{b}$ and $\Pi_{r}$ include all complete graphs $K_{i}$ or trivial graphs $\overline{K_{i}}$ (see Figure 5 for an illustration).

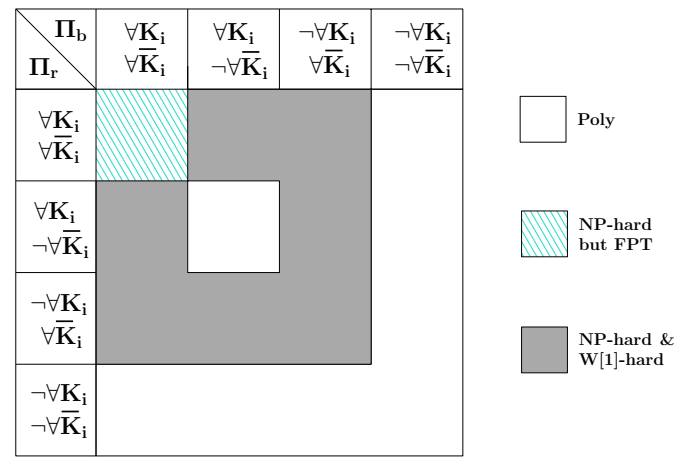

Fig. 5. For a property $\Pi, \forall K_{i}=$ " $\Pi$ includes all complete graphs" and $\neg \forall K_{i}=" \Pi$ excludes some complete graphs". Similar for trivial graphs $\bar{K}_{i}$.

Theorem 6. For hereditary properties $\Pi_{b}$ and $\Pi_{r}$, the complexity of INDUCED $\left(\Pi_{b}, \Pi_{r}\right)$-SUBGRAPH is completely determined as follows:

1. NP-hard and W[1]-hard if one of $\Pi_{b}$ and $\Pi_{r}$ includes all trivial graphs, and the other excludes some complete graphs but includes all trivial graphs or vice versa.

2. NP-hard but FPT if both $\Pi_{b}$ and $\Pi_{r}$ include all complete graphs and all trivial graphs.

3. Polynomial-time solvable if both $\Pi_{b}$ and $\Pi_{r}$ exclude some trivial graphs, or one of $\Pi_{b}$ and $\Pi_{r}$ excludes some complete graphs and some trivial graphs.

We remark that the above theorem implies a complete characterization of the Dual $\Pi$-Subgraph problem, i.e., InducED $(\Pi, \Pi)$-Subgraph, for hereditary $\Pi$ (see the main diagonal of Figure 5).

For the parametric dual problem of Induced $\left(\Pi_{b}, \Pi_{r}\right)$-SubGraph, i.e., deleting $k$ vertices to obtain an induced $\left(\Pi_{b}, \Pi_{r}\right)$-graph, we can easily deduce the following general result on edge-colored multigraphs as a corollary of a well-known result of the first author regarding graph modification problems [3].

Theorem 7. Let $G_{1}, \ldots, G_{t}$ be graphs, and $\Pi_{1}, \ldots, \Pi_{t}$ graph properties characterizable by finite forbidden induced subgraphs. It is FPT to determine whether there are $k$ vertices in $V=\bigcup_{i=1}^{t} V\left(G_{i}\right)$ such that $G\left[V\left(G_{i}\right) \cap V\right]$ is a $\Pi_{i}$-graph for every $1 \leq i \leq t$. 
Edge-bicolored graphs also have close connections with digraphs, and dually connected graphs resemble strongly connected digraphs. We may use ideas in this paper to study subgraph problems on digraphs. In particular, we can easily modify proofs of Theorem 2 and Theorem 3 to obtain the following results for strongly connected subgraphs.

Theorem 8. It is NP-complete and W[1]-hard to determine whether a digraph $G$ contain exactly $k$ vertices $V^{\prime}$ such that $G\left[V^{\prime}\right]$ (resp., $G-V^{\prime}$ ) is strongly connected.

We hope that our work will stimulate further research on simultaneous subgraph problems for edge-bicolored graphs and edge-bicolored multigraphs in general. Indeed, many fundamental and interesting problems are awaiting to be investigated, and we list some open problems here.

Problem 1. Determine whether Dually Connected Deletion is FPT on "dual trees" when blue and red graphs are defined by $G\left[E_{b}\right]$ and $G\left[E_{r}\right]$, instead of $\left(V, E_{b}\right)$ and $\left(V, E_{r}\right)$.

Problem 2. Determine the parameterized complexity of DuAl SEPARATOR and Dual Separator For Two Terminals.

For hereditary properties $\Pi$ not covered by Theorem 7 , the parameterized complexity of DUAL $\Pi$-GRAPH DELETION is open for various fundamental properties $\Pi$. We note that for every property $\Pi$ in the following problem, FPT algorithm exists for turning an uncolored graph into a $\Pi$-graph by deleting $k$ vertices [10 5 17 14 15].

Problem 3. Determine parameterized complexities of DUAL $\Pi$-GRAPH DeLETION for $\Pi$ being acyclic, bipartite, chordal, and planar graphs, respectively.

For a different flavor, we may also consider modifying an edge-bicolored graph into a required graph by edge recoloring. We have obtained some interesting results in connection with dual connectedness and separators, and we will report our findings in a separate paper.

Problem 4. Determine complexities of turning an edge-bicolored graph into a dual $\Pi$-graph for $\Pi$ being acyclic, bipartite, chordal, and planar graphs, respectively, by edge recoloring.

We expect many exciting results concerning simultaneous subgraphs in edgebicolored graphs and edge-colored multigraphs in general, which may also shed light on other graph problems such as problems on digraphs.

Acknowledgement. We are grateful to Michel Habib for bringing our attention to the work on common connected components and common intervals. 


\section{References}

1. Bang-Jensen, J., Gutin, G.: Alternating cycles and paths in edge-coloured multigraphs: a survey. Discrete Mathematics 165, 39-60 (1997)

2. Bui-Xuan, B.M., Habib, M., Paul, C.: Competitive graph searches. Theoretical Computer Science 393(1), 72-80 (2008)

3. Cai, L.: Fixed-parameter tractability of graph modification problems for hereditary properties. Information Processing Letters 58(4), 171-176 (1996)

4. Cai, L., Chan, S.M., Chan, S.O.: Random separation: A new method for solving fixed-cardinality optimization problems. In: Bodlaender, H.L., Langston, M.A. (eds.) IWPEC 2006. LNCS, vol. 4169, pp. 239-250. Springer, Heidelberg (2006)

5. Dehne, F., Fellows, M., Langston, M., Rosamond, F., Stevens, K.: An $O\left(2^{O(k)} n^{3}\right)$ FPT algorithm for the undirected feedback vertex set problem. Theory of Computing Systems 41(3), 479-492 (2007)

6. Downey, R.G., Fellows, M.R.: Parameterized Complexity. Springer, New York (1999)

7. Gai, A.T., Habib, M., Paul, C., Raffinot, M.: Identifying common connected components of graphs. Technical Report RR-LIRMM-03016, LIRMM, Université de Montpellier II (2003)

8. Garey, M.R., Johnson, D.S.: Computers and Intractability: A Guide to the Theory of NP-Completeness. W.H. Freeman, New York (1979)

9. Garey, M.R., Johnson, D.S., Stockmeyer, L.: Some simplified NP-complete graph problems. Theoretical Computer Science 1(3), 237-267 (1976)

10. Guo, J., Gramm, J., Hüffner, F., Niedermeier, R., Wernicke, S.: Compression-based fixed-parameter algorithms for feedback vertex set and edge bipartization. Journal of Computer and System Sciences 72, 1386-1396 (2006)

11. Habib, M., Paul, C.: A survey of the algorithmic aspects of modular decomposition. Computer Science Review 4(1), 41-59 (2010)

12. Kano, M., Li, X.: Monochromatic and heterochromatic subgraphs in edge-colored graphs-a survey. Graphs and Combinatorics 24(4), 237-263 (2008)

13. Khot, S., Raman, V.: Parameterized complexity of finding subgraphs with hereditary properties. Theoretical Computer Science 289(2), 997-1008 (2002)

14. Marx, D.: Chordal deletion is fixed-parameter tractable. Algorithmica 57(4), 747-768 (2010)

15. Marx, D., Schlotter, I.: Obtaining a planar graph by vertex deletion. Algorithmica 62(3-4), 807-822 (2012)

16. Naor, M., Schulman, L.J., Srinivasan, A.: Splitters and near-optimal derandomization. In: Proceedings of the 36th Annual Symposium of Foundations of Computer Science, pp. 182-191 (1995)

17. Reed, B., Smith, K., Vetta, A.: Finding odd cycle transversals. Operations Research Letters 32(4), 299-301 (2004)

18. Uno, T., Yagiura, M.: Fast algorithms to enumerate all common intervals of two permutations. Algorithmica 26(2), 290-309 (2000) 